\title{
Pressure-Induced Enhancement of Optical Properties
}

\section{in Indium Oxide Nanowires}

Pengfei Shen ${ }^{1}$, Douglas A. Blom², Sen Shao ${ }^{3}$, Jinhyuk Choi', Thomas Vog', Yanming $M^{3}$, Lin Wang ${ }^{5}$, Yongjae Lee $e^{* 1,5}$

${ }^{1}$ Department of Earth System Sciences, Yonsei University, Seoul 03722, Korea

${ }^{2}$ NanoCenter \& Department of Chemical Engineering, University of South Carolina, Columbia, SC 29208, USA

${ }^{3}$ State Key Lab of Superhard Materials, College of Physics, Jilin University, Changchun 130012, China

${ }^{4}$ NanoCenter \& Department of Chemistry \& Biochemistry, University of South Carolina, Columbia, SC 29208, USA

${ }^{5}$ Center for High Pressure Science and Technology Advanced Research, Shanghai

201203, China

‘Corresponding Author : yongjaelee@yonsei.ac.kr 
(a)

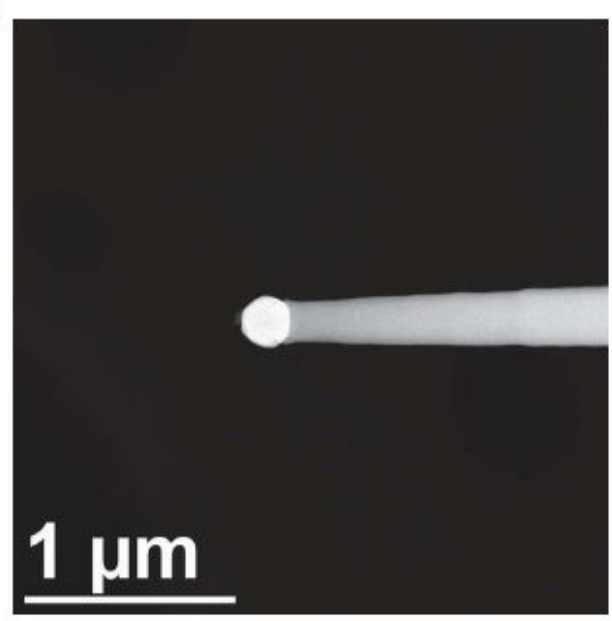

(c)

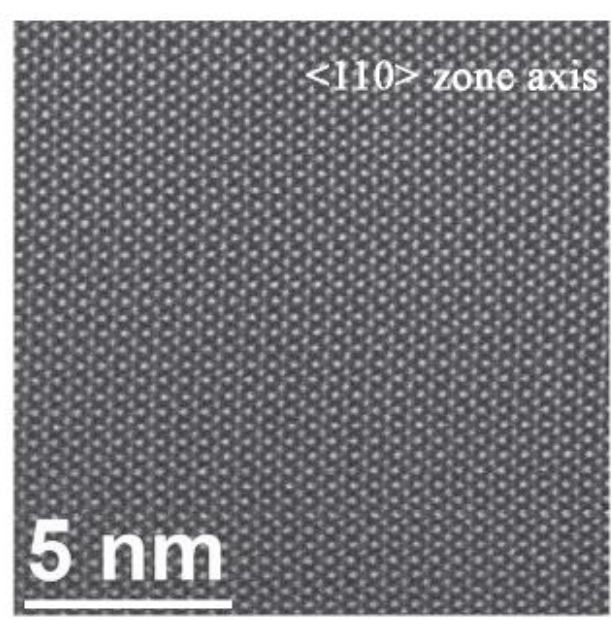

(b)

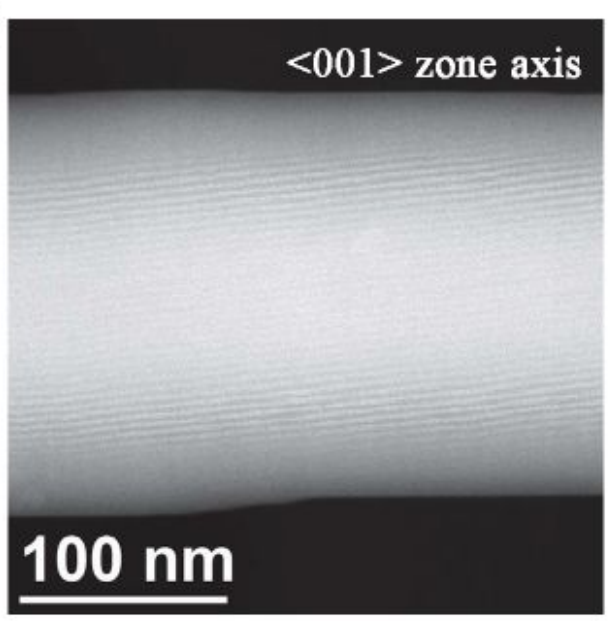

(d)

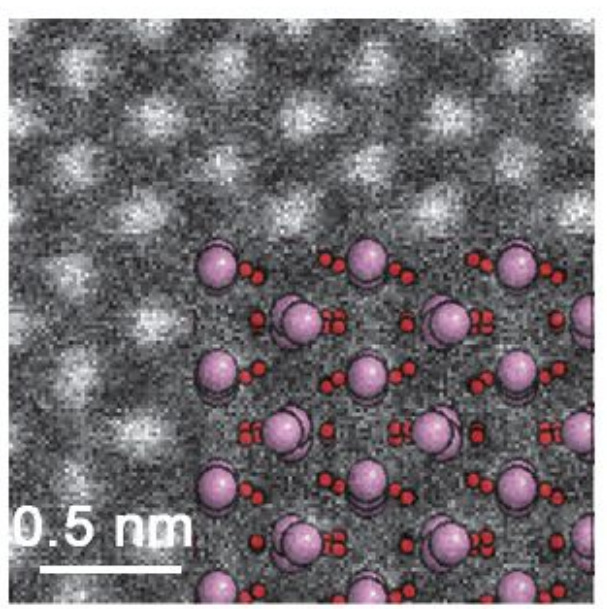

Figure S1. The HAADF STEM images of the typical cubic $\ln _{2} \mathrm{O}_{3} \mathrm{NW}$ in the $<001>$ and $<110>$ zone axis orientation at different scales. 

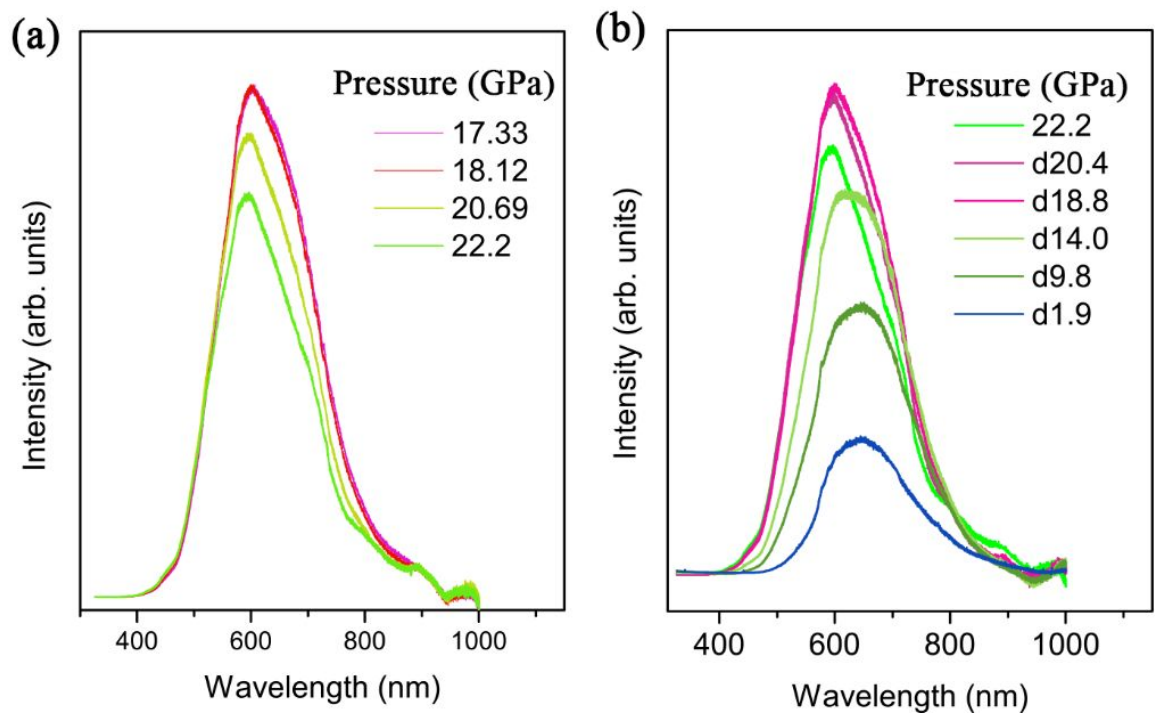

Figure S2. (a) PL emission spectra of NW from 17.33 to $22.2 \mathrm{GPa}$. (b) Representative PL spectra of NW under decompression. 

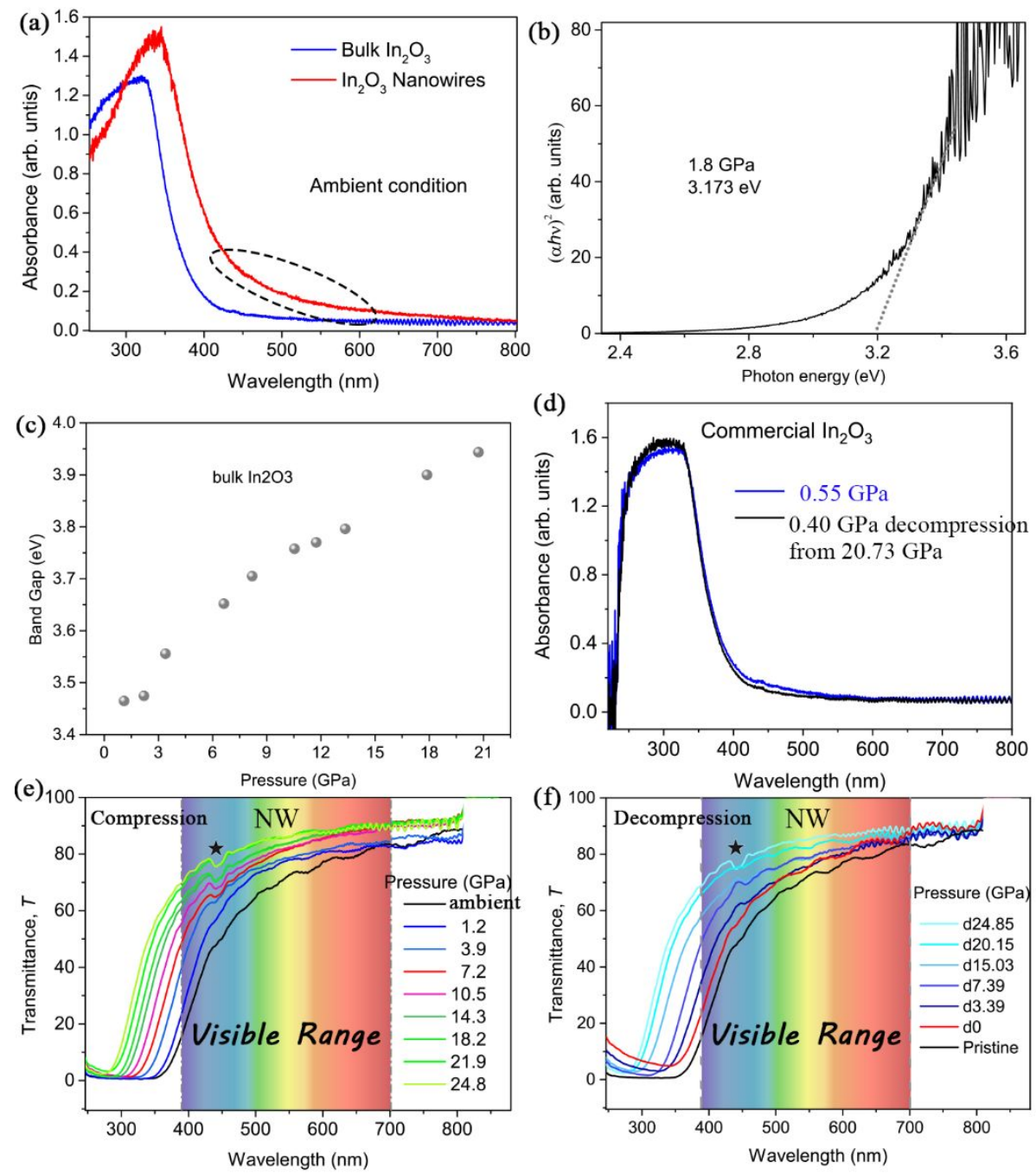

Figure S3. (a) Absorption spectra of bulk and $N W \ln _{2} \mathrm{O}_{3}$ at ambient conditions. The presence of oxygen deficiencies leads to the optical absorption edge shifting toward lower energies which is marked by the dashed area. (b) (c) Evolution of the bandgap of bulk $\operatorname{In}_{2} \mathrm{O}_{3}$ as a function of pressure. (d) Absorbance of spectra of $\ln _{2} \mathrm{O}_{3} \mathrm{NW}$ before and after compression. (e) Optical transmission of NW upon compression. (f) Optical transmittance 
spectra of NW decompression from $24.85 \mathrm{GPa}$ to ambient conditions. Asterisk indicates signal from detector. 

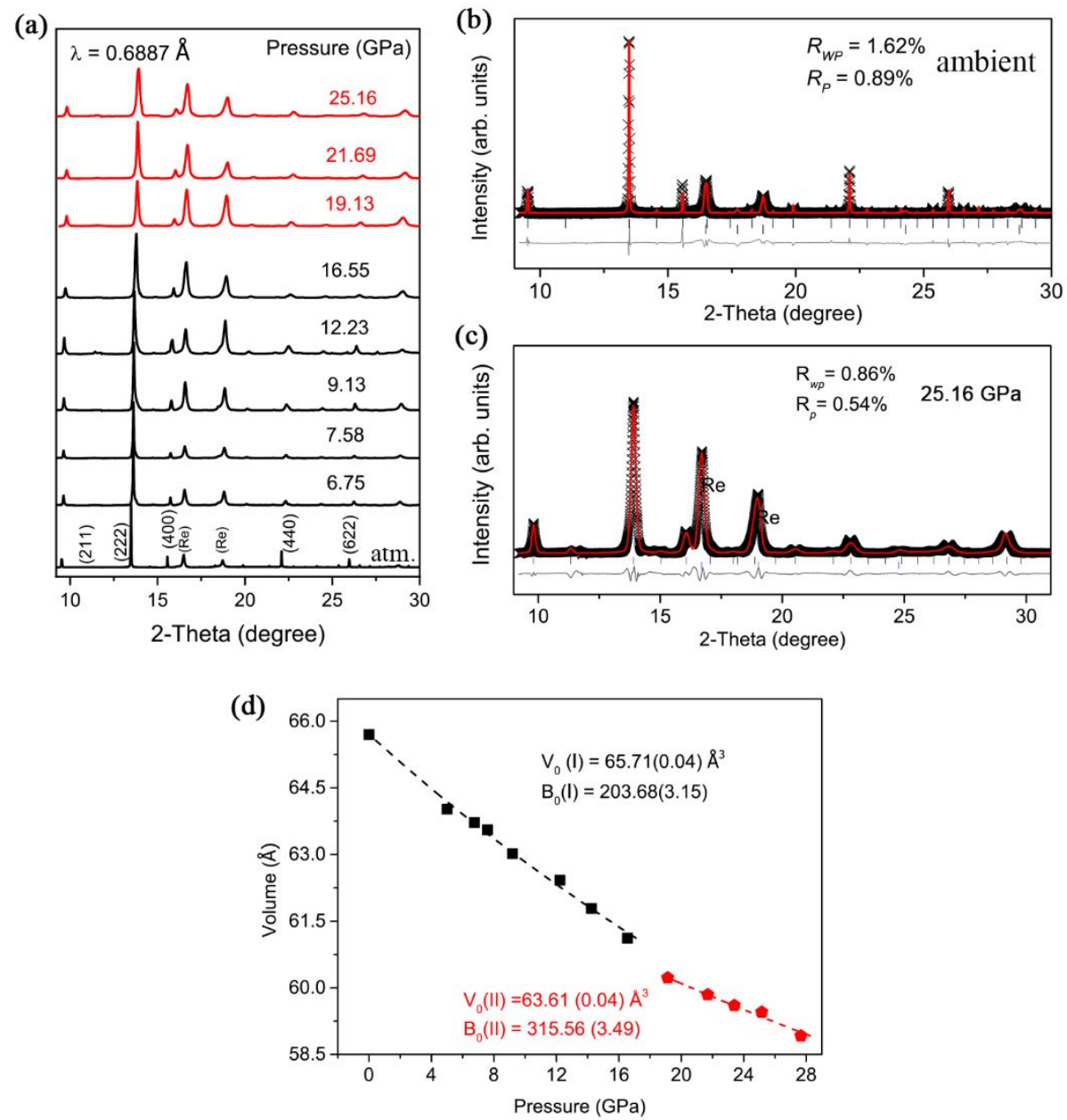

Figure S4. (a) Representative XRD patterns of $\ln _{2} \mathrm{O}_{3} \mathrm{NW}$ at selected pressures. The lattice planes of NW and Rhenium are labeled. (b) and (c) Refinement of XRD data with a bixbyite model in the cubic space group $/ a_{3}^{-}$at ambient pressure and 25.1(6) GPa, respectively. (d) Unit cell volume obtained from Rietveld refinement of NW XRD patterns under pressure. 

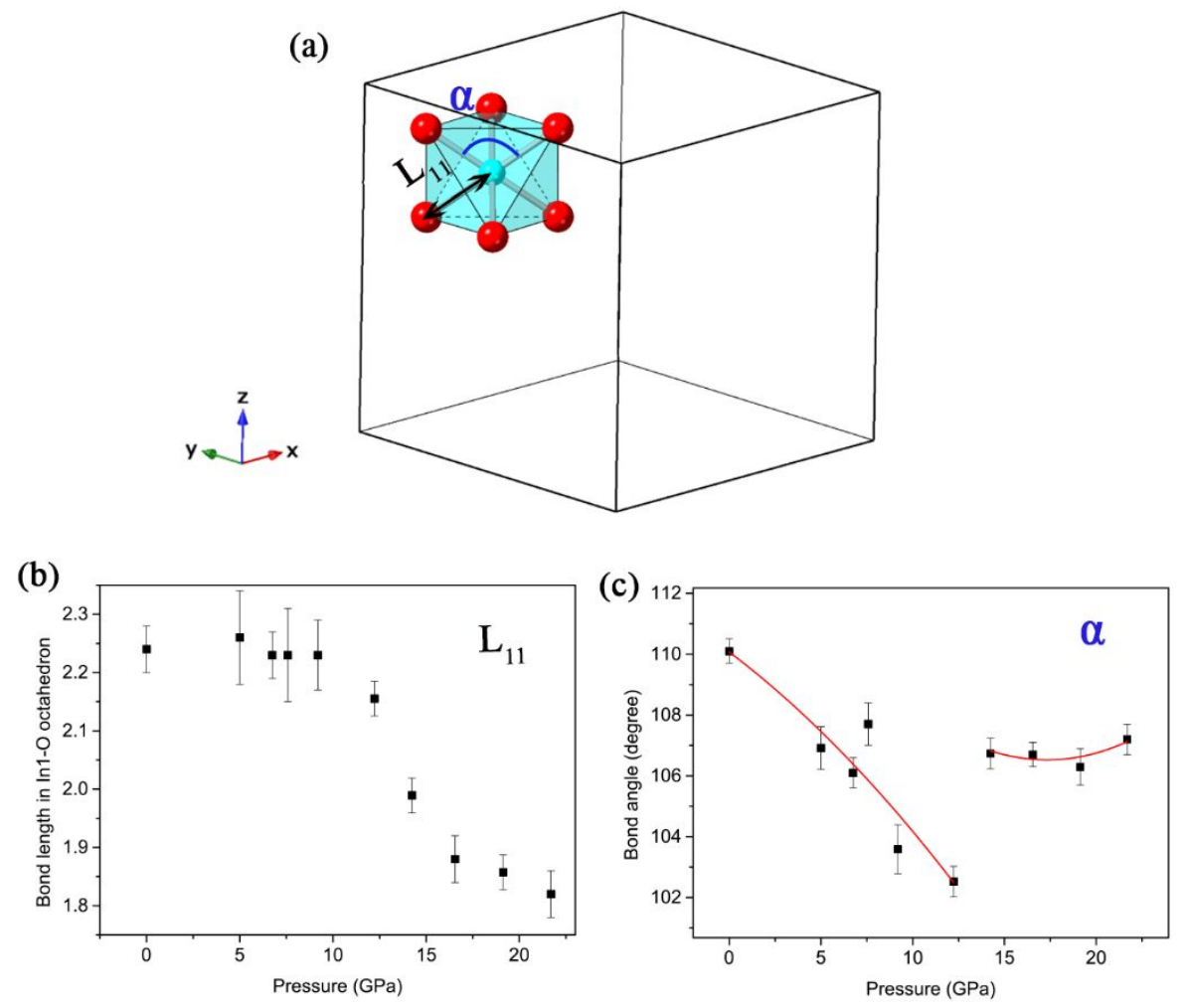

Figure S5. (a) Schematic of an In1-O octahedron in the unit cell. (b) The variation of the bond length of $\ln 1-\mathrm{O}$ as a function of pressure. (c) The variation of the bond angle $\alpha$ as a function of pressure. 

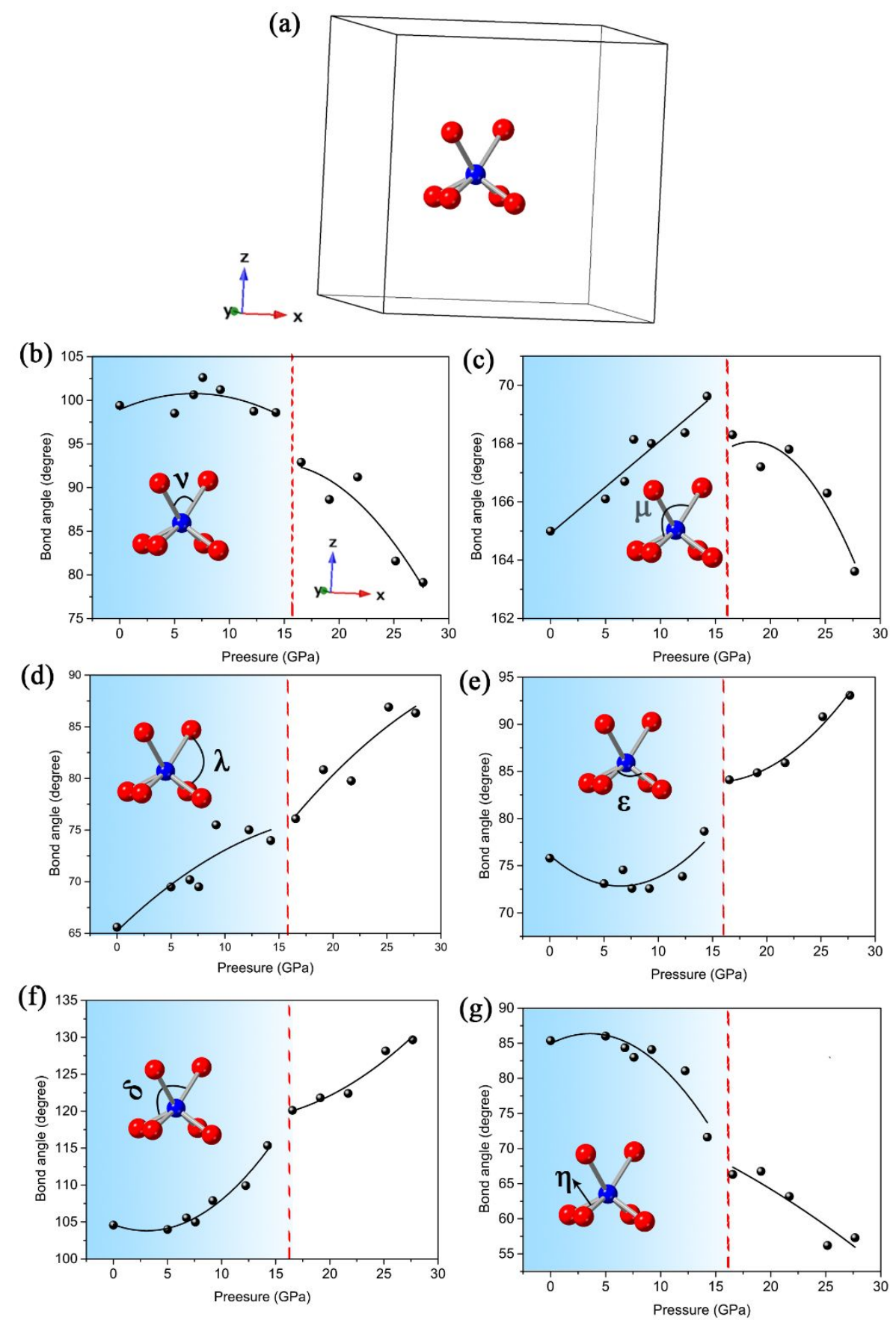
Figure S6. (a) Schematic of In2-O trigonal prism in unit cell. (b)-(g) The variation of the selected bond angles $\angle \mathrm{O}-\operatorname{In} 2-\mathrm{O}$ as a function of pressure. Inset is the schematic diagram of these non-equivalent angles within In2-O trigonal prism. 
(a)

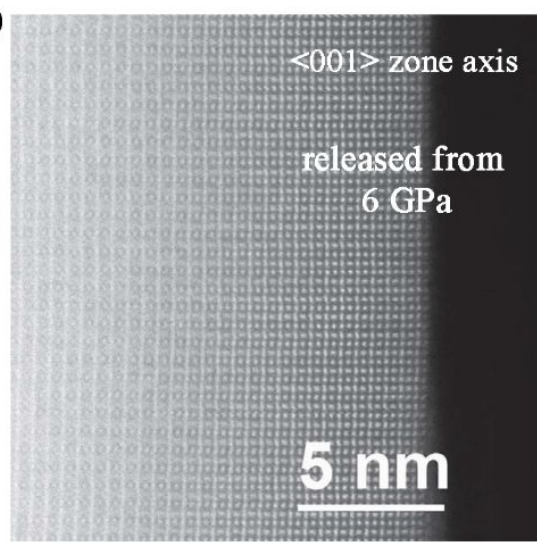

(c)

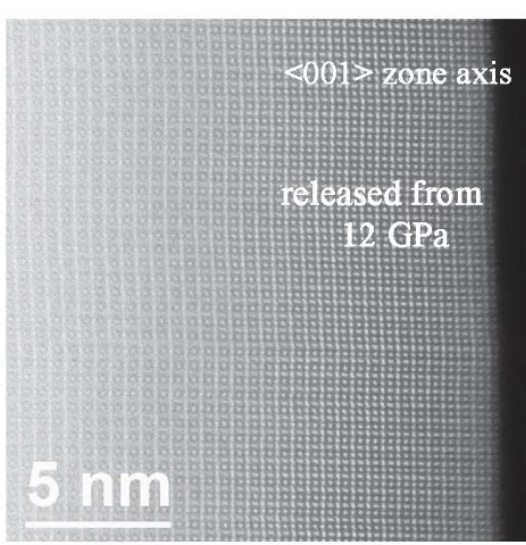

(e)

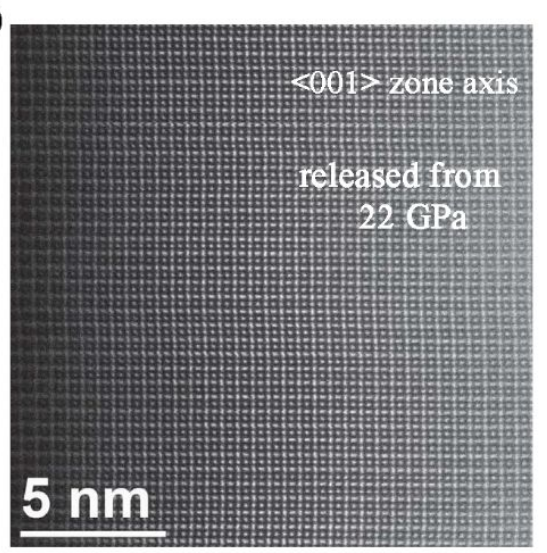

(b)

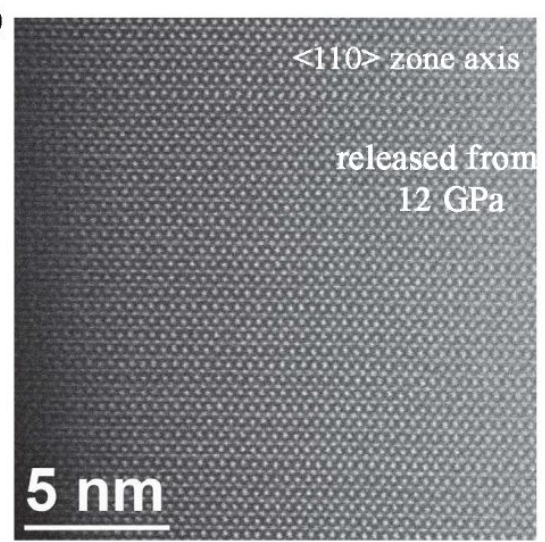

(d)

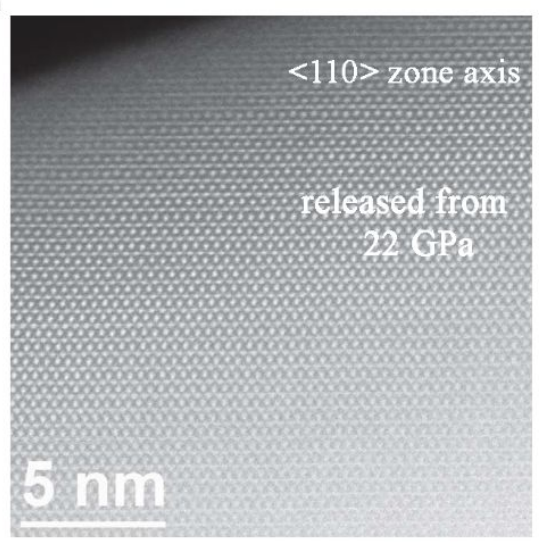

(f)

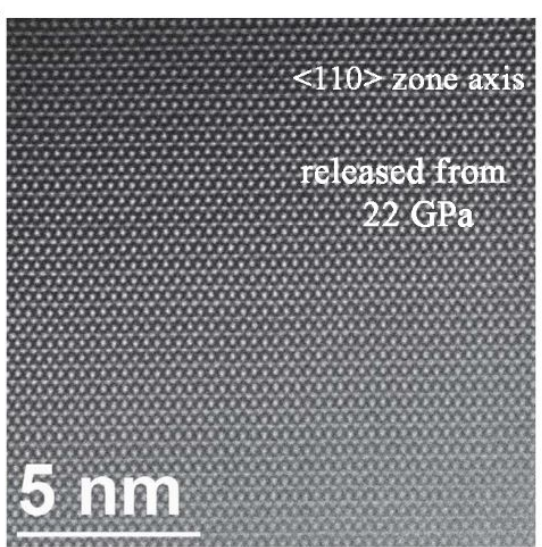

(g)

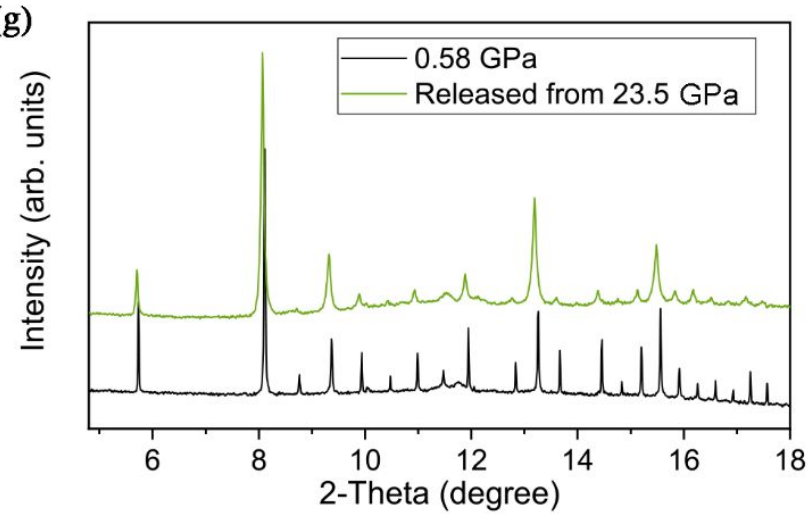


Figure $S 7$ (a)-(f) High-resolution STEM images of $\ln _{2} \mathrm{O}_{3} \mathrm{NW}$ released from different pressures. (g) XRD patterns at $0.58 \mathrm{GPa}$ and released from $23.5 \mathrm{GPa}$ of NW crystal.
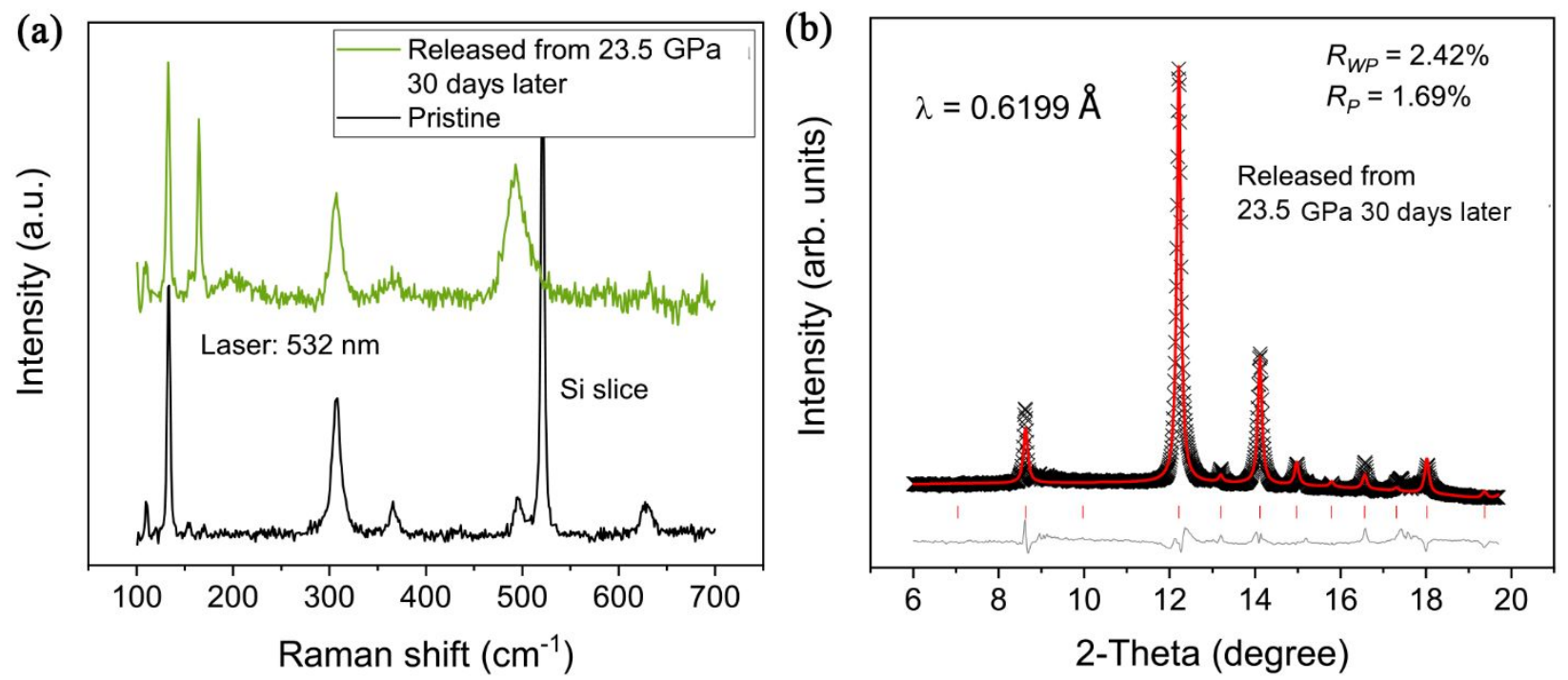

(c)

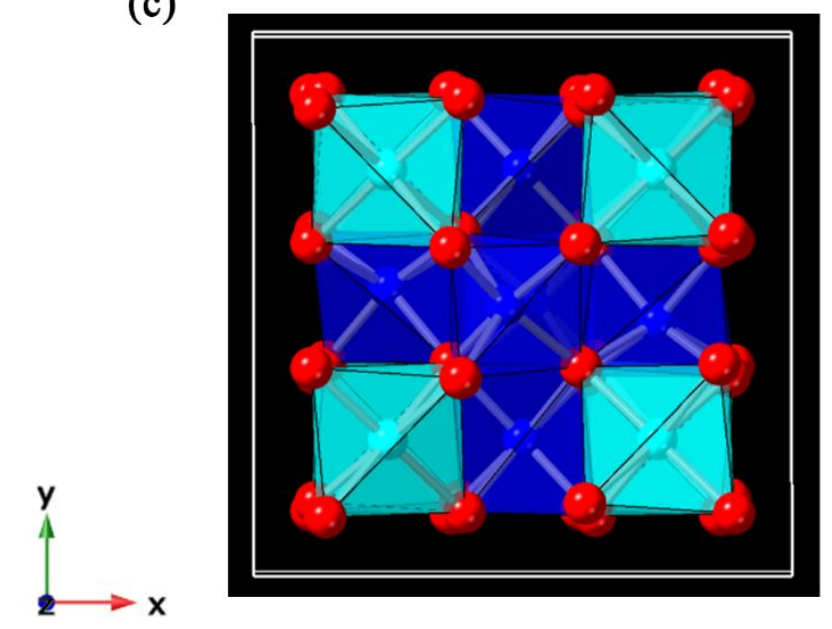

Figure S8. (a) Raman spectra of pristine (black) and released (cyan) sample and (b)

Refinement of XRD spectra of released sample after 30 days. (c) Schematic illustrations of polyhedral distortions in released NW after 30 days. 
(a)

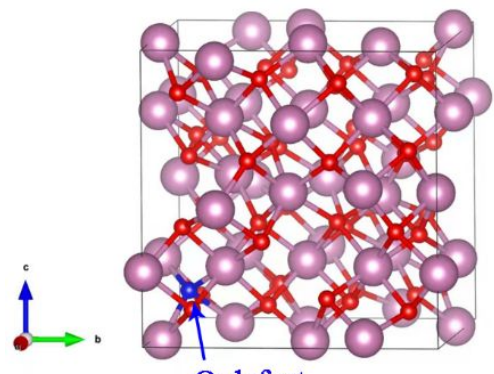

O defect (b)

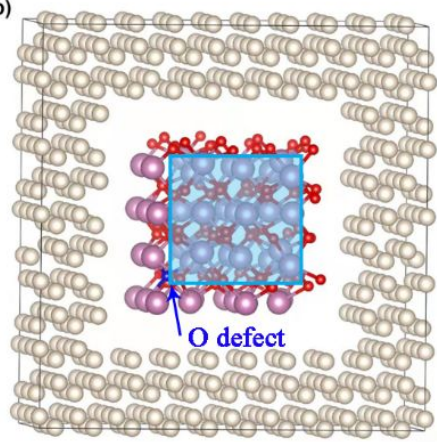

Figure S9. The structures of $\ln _{2} \mathrm{O}_{3}$ bulk and nanowire materials. (a) bulk, (b) nanowire.

Blue atom is the defect $\mathrm{O}$ site in our simulation.
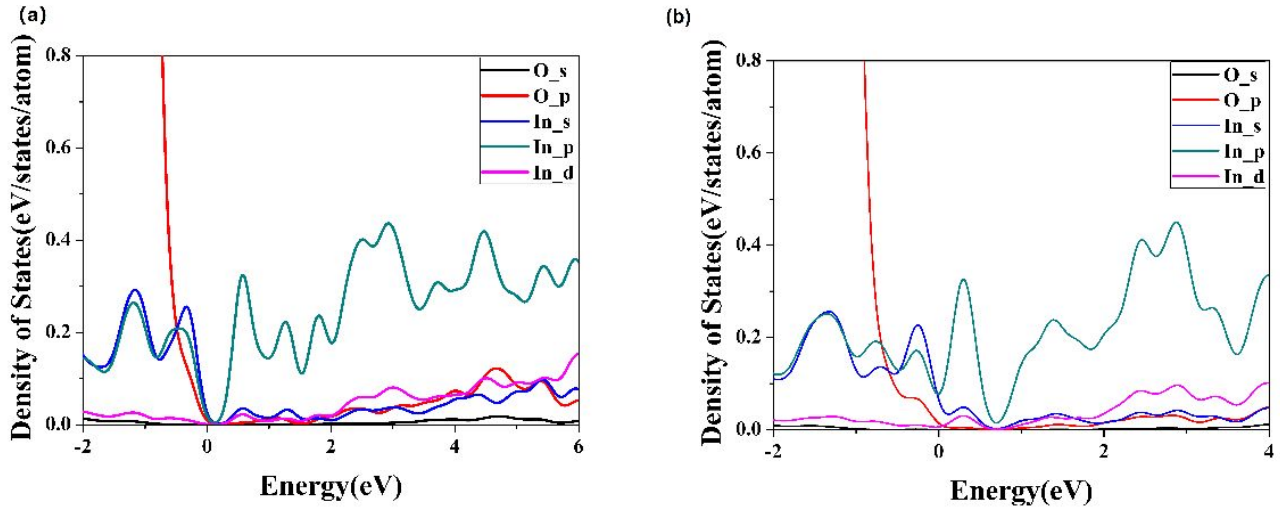

Figure S10. PDOS of surface atoms for defect $\ln _{2} \mathrm{O}_{3}$ nanowire materials (a) at $0 \mathrm{GPa}$, (b) at $14 \mathrm{GPa}$. 
Table S1. The PL position of $\ln _{2} \mathrm{O}_{3} \mathrm{NW}$ in 1931 CIE system

\begin{tabular}{|c|c|c|c|c|}
\hline Pressure & CIE x & CIE y & Peak & Peak Intensity \\
\hline 1.9 & 0.5701 & 0.4189 & 644 & 7749 \\
\hline 3.8 & 0.5549 & 0.4297 & 646 & 8414 \\
\hline 5.58 & 0.5509 & 0.4366 & 644 & 10054 \\
\hline 8.86 & 0.5288 & 0.4436 & 644 & 14874 \\
\hline 10.96 & 0.5245 & 0.4463 & 636 & 20119 \\
\hline 13.16 & 0.5175 & 0.4499 & 638 & 25500 \\
\hline 15.54 & 0.51 & 0.4557 & 607 & 30722 \\
\hline 16.3 & 0.5058 & 0.4578 & 604 & 33591 \\
\hline 20.69 & 0.4832 & 0.4676 & 598 & 30634 \\
\hline 22.2 & 0.476 & 0.4694 & 592 & 26597 \\
\hline
\end{tabular}

Table S2. structural parameters of NW.

\begin{tabular}{|c|c|c|c|c|c|c|c|c|c|}
\hline \multirow[b]{2}{*}{$\begin{array}{l}\text { Pressur } \\
\text { e (GPa) }\end{array}$} & \multicolumn{5}{|c|}{ Octahedron (In1-O) } & \multicolumn{4}{|c|}{ Trigonal prism (In2-O) } \\
\hline & $\mathrm{L}_{11}$ & $\alpha$ & $\beta$ & $\Gamma$ & volume & $\mathrm{L}_{21}$ & $\mathrm{~L}_{22}$ & $\mathrm{~L}_{23}$ & volume \\
\hline 0 & $2.24(4)$ & $110.1(4)$ & $180.0(0)$ & $69.9(4)$ & $\begin{array}{l}11.17(25 \\
)\end{array}$ & $\begin{array}{l}2.020(24 \\
)\end{array}$ & $1.94(2)$ & $\begin{array}{l}2.466(25 \\
)\end{array}$ & $\begin{array}{l}10.52(20 \\
)\end{array}$ \\
\hline 5 & $2.26(8)$ & $106.9(7)$ & $180.0(0)$ & $73.1(8)$ & $\begin{array}{l}12.15(30 \\
)\end{array}$ & $\begin{array}{l}1.931(33 \\
)\end{array}$ & $2.10(4)$ & $2.37(6)$ & $\begin{array}{l}10.45(20 \\
)\end{array}$ \\
\hline 6.75 & $2.23(4)$ & 106.1(5) & $180.0(0)$ & $73.9(7)$ & $\begin{array}{l}12.60(27 \\
)\end{array}$ & $1.91(4)$ & $2.06(4)$ & $2.37(4)$ & $\begin{array}{l}10.48(20 \\
)\end{array}$ \\
\hline 7.58 & $2.23(8)$ & $107.7(7)$ & $180.0(0)$ & $72.3(7)$ & $\begin{array}{l}12.11(15 \\
)\end{array}$ & $\begin{array}{l}1.861(27 \\
)\end{array}$ & $\begin{array}{l}2.178(27 \\
)\end{array}$ & $2.33(5)$ & $\begin{array}{l}10.43(20 \\
)\end{array}$ \\
\hline 9.19 & $2.23(6)$ & $103.6(8)$ & $180.0(0)$ & $76.4(7)$ & $\begin{array}{l}13.20(24 \\
)\end{array}$ & $\begin{array}{l}1.871(20 \\
)\end{array}$ & $\begin{array}{l}2.239(30 \\
)\end{array}$ & $2.26(6)$ & $\begin{array}{l}10.76(32 \\
)\end{array}$ \\
\hline 12.23 & $2.155(3)$ & $102.521(5)$ & $180.0(0)$ & $\begin{array}{l}77.479(2 \\
)\end{array}$ & $\begin{array}{l}12.23(25 \\
)\end{array}$ & $1.83(2)$ & $2.285(3)$ & $2.24(3)$ & $\begin{array}{l}10.94(30 \\
)\end{array}$ \\
\hline 14.24 & $1.99(3)$ & $106.736(5)$ & $180.0(0)$ & $\begin{array}{l}73.264(2 \\
)\end{array}$ & $8.80(28)$ & $1.81(2)$ & $\begin{array}{l}2.211(30 \\
)\end{array}$ & $2.39(3)$ & $\begin{array}{l}10.82(22 \\
)\end{array}$ \\
\hline 16.55 & $1.88(4)$ & $106.7(4)$ & $180.0(0)$ & $73.3(8)$ & $7.43(28)$ & $\begin{array}{l}1.882(32 \\
)\end{array}$ & $\begin{array}{l}2.191(34 \\
)\end{array}$ & $2.45(4)$ & $11.6(2)$ \\
\hline
\end{tabular}




\begin{tabular}{|l|l|l|l|l|l|l|l|l|l|}
\hline 19.13 & $1.86(5)$ & $106.3(6)$ & $180.0(0)$ & $73.7(1)$ & $7.53(29)$ & $1.94(5)$ & $\begin{array}{l}2.191(30 \\
)\end{array}$ & $2.40(6)$ & $\begin{array}{l}12.28(21 \\
)\end{array}$ \\
\hline 21.69 & $1.82(4)$ & $107.2(5)$ & $180.0(0)$ & $72.8(9)$ & $6.61(40)$ & $\begin{array}{l}1.943(34 \\
)\end{array}$ & $\begin{array}{l}2.170(40 \\
)\end{array}$ & $2.42(4)$ & $\begin{array}{l}11.90(33 \\
)\end{array}$ \\
\hline 25.16 & $\begin{array}{l}1.629(28 \\
)\end{array}$ & $98.7(4)$ & $180.0(0)$ & $81.3(10)$ & $5.55(30)$ & $\begin{array}{l}2.143(33 \\
)\end{array}$ & $\begin{array}{l}2.270(40 \\
)\end{array}$ & $\begin{array}{l}2.426(30 \\
)\end{array}$ & $\begin{array}{l}12.77(24 \\
)\end{array}$ \\
\hline 27.67 & $\begin{array}{l}1.624(11 \\
)\end{array}$ & $98.770(4)$ & $180.0(0)$ & $\begin{array}{l}81.230(4 \\
)\end{array}$ & $\begin{array}{l}5.48(31) \\
2.179(15 \\
)\end{array}$ & $\begin{array}{l}2.229(17 \\
)\end{array}$ & $\begin{array}{l}2.486(17 \\
)\end{array}$ & $\begin{array}{l}12.68(25 \\
)\end{array}$ \\
\hline
\end{tabular}

Table S3. Angle parameters of In2-O trigonal prism.

\begin{tabular}{|l|l|l|l|l|l|l|l|l|l|}
\hline Pressure & $\mu$ & $\varepsilon$ & $\vee$ & $\delta$ & $\theta$ & $\eta$ & $\kappa$ & $\lambda$ & $\varphi$ \\
\hline 0 & $165.0(8)$ & $75.8(11)$ & $99.4(11)$ & $104.6(13)$ & $110.6(7)$ & $85.3(15)$ & $124.4(10)$ & $65.6(8)$ & $129.4(12)$ \\
\hline 5 & $166.1(8)$ & $73.1(33)$ & $98.5(25)$ & $104 .(4)$ & $104.4(14)$ & $86 .(5)$ & $135.7(27)$ & $69.5(16)$ & $123.3(20)$ \\
\hline 6.75 & $166.701(0)$ & $74.574(1)$ & $100.626(0)$ & $105.572(1)$ & $103.695(1)$ & $84.366(1)$ & $135.089(0)$ & $70.191(0)$ & $123.060(1)$ \\
\hline 7.58 & $168.1(8)$ & $72.6(30)$ & $102.6(20)$ & $105 .(4)$ & $102.9(16)$ & $83 .(5)$ & $129.2(27)$ & $69.5(18)$ & $122.3(24)$ \\
\hline 9.19 & $168.0(6)$ & $72.6(19)$ & $101.2(16)$ & $107.9(26)$ & $99.9(13)$ & $84.1(31)$ & $135.6(19)$ & $75.5(14)$ & $109.9(22)$ \\
\hline 12.23 & $168.371(0)$ & $73.882(2)$ & $98.732(1)$ & $109.929(3)$ & $99.713(2)$ & $81.055(3)$ & $133.938(1)$ & $75.016(2)$ & $112.784(2)$ \\
\hline 14.24 & $169.623(0)$ & $78.644(2)$ & $98.582(1)$ & $115.346(3)$ & $100.599(2)$ & $71.621(4)$ & $124.037(2)$ & $73.993(1)$ & $113.835(2)$ \\
\hline 16.55 & $168.3(12)$ & $84.1(17)$ & $92.9(16)$ & $120.1(19)$ & $98.3(8)$ & $66.3(24)$ & $124.1(16)$ & $76.1(8)$ & $115.1(19)$ \\
\hline 19.13 & $167.203(0)$ & $84.855(2)$ & $88.636(1)$ & $121.799(2)$ & $94.650(1)$ & $66.739(3)$ & $129.711(1)$ & $80.842(1)$ & $110.464(1)$ \\
\hline 21.69 & $167.8(15)$ & $85.9(17)$ & $91.2(17)$ & $122.4(18)$ & $95.7(7)$ & $63.2(24)$ & $121.9(17)$ & $79.8(8)$ & $115.0(20)$ \\
\hline 25.16 & $166.3(13)$ & $90.8(14)$ & $81.6(19)$ & $128.1(11)$ & $91.2(6)$ & $56.2(14)$ & $126.7(12)$ & $86.9(5)$ & $105.5(19)$ \\
\hline 27.67 & $163.618(1)$ & $93.070(4)$ & $79.137(2)$ & $129.643(5)$ & $90.456(2)$ & $57.302(6)$ & $130.459(2)$ & $86.328(2)$ & $108.994(3)$ \\
\hline
\end{tabular}

